\begin{tabular}{|c|}
\hline $\begin{array}{r}\text { PRAMANA } \\
- \text { journal of } \\
\text { physics }\end{array}$ \\
\hline
\end{tabular}

\title{
Dynamo in protostars
}

\author{
MAHENDRA K VERMA ${ }^{1}$, BIDYA BINAY KARAK ${ }^{2,3}$ \\ and ROHIT KUMAR ${ }^{1, *}$ \\ ${ }^{1}$ Department of Physics, Indian Institute of Technology, Kanpur 208 016, India \\ ${ }^{2}$ Department of Physics, Indian Institute of Science, Bangalore 560 012, India \\ ${ }^{3}$ Nordita KTH Royal Institute of Technology and Stockholm University, Roslagstullsbacken 23, \\ SE-106 91 Stockholm, Sweden \\ *Corresponding author. E-mail: rohitkr@iitk.ac.in
}

MS received 13 April 2013; revised 16 September 2013; accepted 3 October 2013

DOI: 10.1007/s12043-013-0628-y; $\boldsymbol{e}$ Publication: 23 November 2013

\begin{abstract}
In this paper, we estimate the magnetic Reynolds number of a typical protostar before and after deuterium burning, and claim for the existence of dynamo process in both the phases, because the magnetic Reynolds number of the protostar far exceeds the critical magnetic Reynolds number for dynamo action. Using the equipartition of kinetic and magnetic energies, we estimate the steady-state magnetic field of the protostar to be of the order of kilogauss, which is in good agreement with observations.
\end{abstract}

Keywords. Protostar; magnetic field generation; dynamo.

PACS Nos 95.30.Qd; 97.21.+a; 47.27.Jv

\section{Introduction}

Magnetic field is omnipresent in the Universe. It is found in many stars, planets, galaxies, interstellar medium, etc., and it is believed to induce inflow of matter or accretion in astrophysical disc and immersed cloud, collimated jets and outflows, magnetic breaking, and protostellar winds. The magnetic field plays a crucial role in all stages of the evolution of a protostar. In this paper we shall address an important question related to protostars: when does the magnetic field appear in protostars and what is the magnitude of the magnetic field, if present?

Presence of X-ray in the protostar regions suggests a strong magnetic field in its central region [1,2]. Also, the detection of polarized synchrotron emission arising from protostar jets [3] and the measurements from Zeeman broadening of photospheric lines [4-8] strongly support the existence of the magnetic field in the protostars. Several questions on protostars are: whether the magnetic field arises due to the molecular cloud of the protostar formation region or due to the self-induction or dynamo mechanism? How does 
the magnetic field sustain itself and compensate its removal due to the outflows, or its dissipation as ohmic heating? Does the dynamo mechanism, if any, stop after the formation of the protostars?

Machida et al [9] and Sur et al [10] argue that the dynamo mechanism can amplify the initial magnetic field of the star-forming regions. On the other hand, Tan and Blackman [11] suggest that dynamo amplification of the primordial magnetic field is possible in protostellar disc, and they propose that the induced magnetic field is helical. In these works, the generation mechanism for the magnetic field involves several mechanisms, e.g., convection, rotation, etc. However, there is no substantive statement in the literature on the necessity of dynamo process in protostars. In the present paper we claim that the protostars are magnetic because its magnetic Reynolds number to trigger the dynamo process is far above the critical magnetic Reynolds number observed in laboratory experiments and numerical simulations. The equipartition of kinetic and magnetic energies yields the magnetic field of the protostar to be of the order of kilogauss, which is in good agreement with those observed in nature. We present these arguments in the next two sections of the paper, and conclude in the last section.

\section{Critical values of magnetic Reynolds number for dynamo}

The fluid velocity $\mathbf{u}$ and the magnetic field $\mathbf{B}$ in a dynamo mechanism are governed by the magnetohydrodynamic (MHD) equations:

$$
\begin{aligned}
& \rho\left\{\partial_{t} \mathbf{u}+(\mathbf{u} \cdot \nabla) \mathbf{u}\right\}=-\nabla p+(\mathbf{B} \cdot \nabla) \mathbf{B}+v \nabla^{2} \mathbf{u}+\mathbf{F}, \\
& \partial_{t} \mathbf{B}+(\mathbf{u} \cdot \nabla) \mathbf{B}=(\mathbf{B} \cdot \nabla) \mathbf{u}+\eta \nabla^{2} \mathbf{B} \\
& \nabla \cdot \mathbf{B}=0
\end{aligned}
$$

where $\mathbf{u}, \mathbf{B}, \mathbf{F}, p, \rho$ respectively represent the velocity field, magnetic field, external forcing, total pressure (hydrodynamic + magnetic), and density. The parameters $v$ and $\eta$ are the kinematic viscosity and magnetic diffusivity respectively. Under the incompressibility approximation, the density of the fluid is assumed to be a constant $[12,13]$. Note that only the velocity field is being forced in the above equation.

The equation for the magnetic energy is

$$
\partial_{t} \int \mathrm{d} \mathbf{x} \frac{B^{2}}{2}=-\int \mathrm{d} \mathbf{x} \mathbf{B} \cdot(\mathbf{u} \cdot \nabla) \mathbf{B}+\int \mathrm{d} \mathbf{x} \mathbf{B} \cdot(\mathbf{B} \cdot \nabla) \mathbf{u}+\int \mathrm{d} \mathbf{x} \eta \mathbf{B} \cdot \nabla^{2} \mathbf{B}
$$

In the above equation, the first term in the right-hand side (RHS) vanishes because

$$
\int \mathrm{d} \mathbf{x} \mathbf{B} \cdot(\mathbf{u} \cdot \nabla) \mathbf{B}=\int \mathrm{d} \mathbf{x} \nabla \cdot\left(\mathbf{u} \frac{B^{2}}{2}\right)=\int \mathrm{d} \mathbf{S} \cdot\left(\mathbf{u} \frac{B^{2}}{2}\right)=0 .
$$

The third term in the RHS represents the loss of magnetic energy via Joule dissipation, and it is negative for finite $\eta$. The second term in the RHS represents the rate of energy transfer from the velocity field to magnetic field $(U 2 B)$ [14]. For the magnetic field to grow, the $U 2 B$ energy transfer must be positive and larger than the Joule dissipation. Dynamo mechanism is said to occur when the magnetic energy reaches a finite value asymptotically, that is as $t \rightarrow \infty$. Under the steady state, the two rates would be statistically equal. 


\section{Dynamo in protostars}

For the magnetic energy to grow and reach a steady state, a comparison of the aforementioned two terms ( $U 2 B$ and the Joule dissipation) suggests that

$$
\left(\int \mathrm{d} \mathbf{x B} \cdot(\mathbf{B} \cdot \nabla) \mathbf{u}\right)>\left(-\int \mathrm{d} \mathbf{x} \eta \mathbf{B} \cdot \nabla^{2} \mathbf{B}\right) .
$$

Using dimensional analysis, we can deduce that the magnetic Reynolds number

$$
\mathrm{Rm}=\frac{U L}{\eta}>1
$$

The minimum magnetic Reynolds number, called the critical magnetic Reynolds number $\mathrm{Rm}_{\mathrm{c}}$, can be larger than the one due to geometrical consideration or other factors like magnetic Prandtl number (Pm, defined as $v / \eta)$, rotation, etc. Yet, we can argue that $\mathrm{Rm}_{\mathrm{c}}$ is of the order of unity for all dynamo processes; this limit has been consistently observed in a number of laboratory experiments and numerical simulations.

Among laboratory experiments, Gailities (Riga experiment) et al [15], Stieglitz and Müller (Karlsruhe experiment) [16], and Monchaux et al (VKS experiment) [17] observed self-generated magnetic fields in their experimental set-ups. Liquid sodium, whose magnetic Prandtl number is $\sim 10^{-5}$, was used as the operating fluid in the aforementioned experiments. The critical magnetic Reynolds numbers for the above experiments were greater than 10; for example, $\mathrm{Rm}_{\mathrm{c}} \approx 30$ for Monchaux et al's experiment, commonly referred to as VKS experiment [17].

A large number of high-resolution simulations were performed to study the dynamo transition. Most researchers used pseudospectral method to solve the MHD equations for various forcing in a box geometry. The range of magnetic Prandtl number used so far is from $10^{-2}$ to $10^{2}$. Note that numerical simulations of very large Pm or very small Pm dynamo are difficult due to their requirements of high resolutions. Notably, Schekochihin et al [18,19], Ponty et al [20], and Iskakov et al [21] simulated dynamo and studied variation of $\mathrm{Rm}_{\mathrm{c}}$ as a function of magnetic Prandtl number. They observed dynamo process for both small and large Prandtl numbers, with the range of $\mathrm{Rm}_{\mathrm{c}}$ between 10 and 500. The $\mathrm{Rm}_{\mathrm{c}}$ for lower Prandtl numbers tends to be higher in most of the simulations. Recently, Yadav et al $[22,23]$ studied Taylor-Green dynamo and observed the $\mathrm{Rm}_{\mathrm{c}}$ to be around 10 . In figure 1, we plot $\mathrm{Rm}_{\mathrm{c}}$ for the aforementioned experiments and numerical simulations. We can conclude from these results and the aforementioned phenomenological arguments relating the nonlinear term to the dissipative term that $\mathrm{Rm}_{\mathrm{c}}$ varies with geometry, forcing, and Prandtl number, but it remains bounded between 1 and 500. Hence, if the protostars have $\mathrm{Rm}>\mathrm{Rm}_{\mathrm{c}}$, then dynamo must be active in the protostar. This is what we will estimate in the next section.

\section{Magnetic Reynolds number and dynamo action in the protostar}

In this section we shall discuss the dynamo process in the protostar before and after deuterium burning. First, we focus on a typical protostar before deuterium burning. Temperature of the protostar at this stage may be around $10^{4} \mathrm{~K}$, its radius around 100 solar radii, and its mass around two solar mass. The kinematic viscosity and the resistivity of 


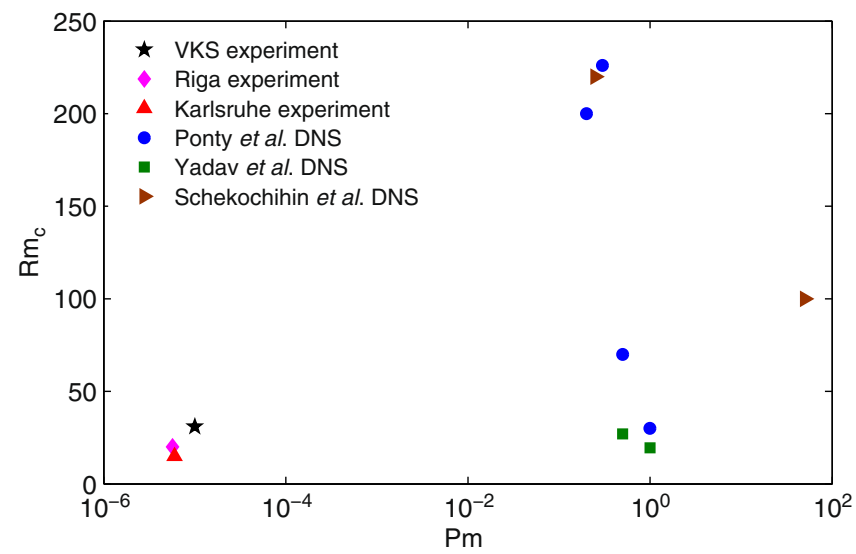

Figure 1. Plot of the critical magnetic Reynolds number $\left(\mathrm{Rm}_{\mathrm{c}}\right)$ vs. magnetic Prandtl number $(\mathrm{Pm})$. The plot exhibits data of Riga experiment [15], Karlsruhe experiment [16], VKS experiment [17], and numerical data of Ponty et al (direct numerical simulation (DNS)) [20], Yadav et al (DNS) [22,23], and Schekochihin et al (DNS) [18,19].

the gas can be estimated using the Spitzer formula (see e.g., Schekochihin et al [24] or Choudhuri [12]) that provides

$$
\begin{aligned}
& v \sim 2.21 \times 10^{-15} T^{5 / 2} /(4 \rho), \\
& \eta \sim 4 \pi^{3 / 2} m_{\mathrm{e}}^{1 / 2} e^{2} c^{2} /\left(2 \times\left(2 k_{\mathrm{B}} T\right)^{3 / 2} \times 0.6\right),
\end{aligned}
$$

where $\rho$ is the density of the medium, $T$ is the temperature in Kelvin, $k_{\mathrm{B}}$ is the Boltzmann's constant, $m_{\mathrm{e}}$ and $e$ are the mass and charge of an electron, and $c$ is the speed of light. Note that the above formulas are in CGS units. We substitute the aforementioned parameters, $T=10^{4} \mathrm{~K}$ and the average density $\rho \approx 3 \times 10^{-6} \mathrm{~g} / \mathrm{cm}^{3}$, in the above formulas. We take the radius of the protostar to be 100 times the solar radius, which gives the radius of a typical protostar to be $7 \times 10^{12} \mathrm{~cm}$. We estimate the length of the large eddies to be one-tenth of the protostar radius, which provides $L \approx 7 \times 10^{11} \mathrm{~cm}$. The large-scale velocity $U$ is estimated to be one-tenth of the sound speed. Consequently,

$$
\begin{aligned}
& v \approx 2 \mathrm{~cm}^{2} / \mathrm{s}, \\
& \eta \approx 3 \times 10^{7} \mathrm{~cm}^{2} / \mathrm{s}, \\
& U \approx 10^{5} \mathrm{~cm} / \mathrm{s}, \\
& \mathrm{Rm} \approx 3 \times 10^{9} \\
& \mathrm{Pm} \approx 10^{-7}
\end{aligned}
$$

The magnetic Reynolds number $\mathrm{Rm}$ is much greater than the estimated critical magnetic Reynolds number (10-500) using the laboratory experimental and numerical data. Hence we expect dynamo to be active in typical protostars before the deuterium burning 


\section{Dynamo in protostars}

stage. The background galactic magnetic field or the magnetic field amplified during the formation of protostars can act as the seed magnetic field for this process.

The saturated or the steady-state magnetic field of the protostar can be estimated easily using the equipartition of kinetic and magnetic energies, i.e., $B^{2} / 4 \pi \sim \rho U^{2}$, which gives the average magnetic field at this stage to be around $700 \mathrm{G}$. Note that an approximate equipartition of kinetic and magnetic energies in the steady state has been observed in a large number of systems (e.g., solar wind) and numerical simulations, and hence it is a robust assumption for estimating the steady-state magnetic field. Numerical simulations also reveal that saturation of the magnetic field takes around 10 eddy turnover time. Hence we expect that the time taken for the magnetic field of the protostar to reach a steady state would be $\sim 10 L / U$, which is $\sim 686$ terrestrial days.

Now, let us study the possibility of dynamo process in the protostar post deuterium burning. At this stage, the temperature of a typical protostar is $\sim 10^{6} \mathrm{~K}$, and its radius is that of a typical main sequence star. For simplicity, we take the radius of the postdeuterium burning protostar to be the solar radius and mass to be twice the solar mass. If we take the large-length scale $L$ to be one tenth of the solar radius and time period of the large eddies to be the rotation time period of the protostar, which is $\sim 20$ terrestrial days, we get

$$
\begin{aligned}
& v \approx 0.2 \mathrm{~cm}^{2} / \mathrm{s}, \\
& \eta \approx 3 \times 10^{4} \mathrm{~cm}^{2} / \mathrm{s}, \\
& U \approx 4 \times 10^{3} \mathrm{~cm} / \mathrm{s}, \\
& \mathrm{Rm} \approx 1 \times 10^{9}, \\
& \mathrm{Pm} \approx 8 \times 10^{-6} .
\end{aligned}
$$

Again, since $\mathrm{Rm}>\mathrm{Rm}_{\mathrm{c}}$, we expect dynamo to be active in the protostar after the deuterium burning phase. The magnetic field generated in the pre-deuterium burning stage will act as the seed magnetic field. Equipartition of the kinetic and magnetic energies gives the average magnetic field to be of the order of $2 \times 10^{4} \mathrm{G}$, and it will take around 200 terrestrial days for the magnetic field to reach saturation. Note that our simple estimate of the protostar magnetic field is in general agreement with many observational measurements of Zeeman broadening [4-8], which reveal the existence of magnetic field of kilogauss strength. The high field thus generated can produce X-ray activity, jets, outflows, and other magnetic effects. We remark that the above estimates are in good agreement with the observed parameters of the Sun.

\section{Conclusions}

In this paper, we estimate the magnetic Reynolds number Rm of a typical protostar before and after deuterium burning. We show that the $\mathrm{Rm}$ in both the phases are far greater than the critical magnetic Reynolds number $\mathrm{Rm}_{\mathrm{c}}$, which can be between 10 and 500, an estimate based on dimensional analysis, laboratory experiments, and numerical simulations. Thus, we claim that the dynamo mechanism is active in protostar in both the phases. We 
also estimate the steady-state magnetic field of the protostar in these two phases using the equipartition of kinetic and magnetic energies, and find it to be of the order of a kilogauss, which is in very good agreement with several astronomical observations [4-8].

Our arguments on protostar dynamo is quite robust, and it is independent of the mechanism invoked for the process, e.g., convection, rotation, $\alpha-\omega$, etc. It must be however kept in mind that the actual magnetic field of the star would depend on the details of the dynamo process. We provide an argument in favour of the dynamo mechanism, as well as an estimate of the strength of the magnetic field, in protostar.

It is important however to note that the present observations on the magnetic field are not very conclusive, since the observations depend quite critically on the measurement techniques [1-8]. Observation of the magnetic field of a protostar in pre-deuterium burning phase is very difficult since a protostar is embedded in deep clouds, and optical measurements are difficult for these systems. Therefore, most of the measured values of a protostar have wide uncertainties. However, the measurements of the physical quantities of the protostar in the post-deuterium burning phase is more reliable; we have used observationally motivated values of the radius, mass, temperature, and rotation period in our estimation (e.g., [8,25]). It is hoped that future astronomical observations would provide stronger evidences that would help us validate the arguments on the dynamo processes in protostars.

\section{Acknowledgements}

The authors thank the anonymous referee for careful reading and suggestions which helped us to improve the manuscript. They are also grateful to Owen Mathews, Prateek Sharma, Dinshaw Balsara, Banibrata Mukhopadhyay, H C Bhatt and Sagar Chakraborty for valuable suggestions and comments at various stages.

\section{References}

[1] T Montmerle, N Grosso, Y Tsuboi and K Koyama, Astrophys. J. 532, 1097 (2000)

[2] K Hamaguchi et al, in: Proceedings of the 'The X-ray Universe 2005' edited by A Wilson (El Escorial, Madrid, Spain, 26-30 September 2005) ESA SP-604, Volume 1, Noordwijk: ESA Publications Division, ISBN 92-9092-915-4, 2006, pp. 33-38

[3] C Carrasco-González et al, Science 330, 1209 (2010)

[4] C M Johns-Krull, J A Valenti and C Koresko, Astrophys. J. 516, 900 (1999)

[5] E W Guenther, H Lehmann, J P Emerson and J Staude, Astron. Astrophys. 341, 768 (1999)

[6] H Yang, C M Johns-Krull and J A Valenti, Astrophys. J. 635, 466 (2005)

[7] J Bouvier, S H P Alencar, T J Harries, C M Johns-Krull and M M Romanova, Protostars and planets $V$ edited by B Reipurth, D Jewitt and K Keil (University of Arizona Press, Tucson, 2007) pp. 479-494

[8] J Bouvier et al, Astron. Astrophys. 318, 495 (1997)

[9] M N Machida, S Inutsuka and T Matsumoto, Astrophys. J. 670, 1198 (2007)

[10] S Sur, F Schleicher, R G Dominik, R Banerjee and R S Klessen, Mon. Not. R. Astron. Soc. 423, 3148 (2012)

[11] J C Tan and E G Blackman, Astrophys. J. 603, 401 (2004)

[12] A R Choudhuri, The physics of fluids and plasmas: An introduction for astrophysicists (Cambridge University Press, 1998) 
[13] P H Roberts and G A Glatzmaier, Rev. Mod. Phys. 72, 1081 (2000)

[14] M K Verma, Phys. Rep. 401, 229 (2004)

[15] A Gailitis et al, Phys. Rev. Lett. 86, 3024 (2001)

[16] R Stieglitz and U Müller, Phys. Fluids 13, 561 (2001)

[17] R Monchaux et al, Phys. Rev. Lett. 98, 044502 (2007)

[18] A Schekochihin et al, Astrophys. J. 612, 276 (2004)

[19] A Schekochihin et al, Phys. Rev. Lett. 92, 054502 (2004)

[20] Y Ponty et al, Phys. Rev. Lett. 94, 164502 (2005)

[21] A B Iskakov et al, Phys. Rev. Lett. 98, 208501 (2007)

[22] R Yadav et al, Europhys. Lett. 91, 69001 (2010)

[23] R Yadav et al, Phys. Rev. E 85, 036301 (2012)

[24] A A Schekochihin, S C Cowley, R M Kulsrud, G W Hammett and P Sharma, Astrophys. J. 629, 139 (2005)

[25] P Andre and T Montmerle, Astrophys. J. 420, 837 (1994) 